\title{
Depressão pós-parto: incidência e fatores de risco associados
}

\section{Postpartum depression: incidence and risk factors associate}

\author{
Ana Carolina Emerenciano Guedes', Cinthia Tiemi Kami', \\ Laura Kolb de Vargas Cavalli', Stephanie Kosmos Nicolaou', \\ Válery Baggio Hess ${ }^{1}$, Eliane Mara Cesário Pereira Maluf ${ }^{2}$
}

Guedes ACE, Kami CT, Cavalli LKV, Nicolaou SK, Hess VB, Maluf EMCP. Depressão pós-parto: incidência e fatores de risco associados/Postpartum depression: incidence and risk factors associate. Rev Med (São Paulo). 2011 jul.set.;90(3):149-54.

RESUMO: As doenças psiquiátricas do pós-parto foram reconhecidas como transtorno específico há pouco tempo. São, por isso, pouco pesquisadas e de escasso conhecimento. Contudo, mostram-se de identificação importante pela morbidade e frequência com que acometem as puérperas. A prevalência de depressão pós-parto (DPP) é de 10-15\%, com estudos apontando para $22 \%$. Sua etiologia é multifatorial e inclui fatores biológicos, psicológicos e sociais, podendo durar até um ano após o parto. Buscou-se avaliar a incidência de DPP em puérperas no primeiro ano de pós-parto na cidade de Curitiba - PR, tentando ainda identificar as mudanças de humor ocorridas no pós-parto e os principais fatores de risco associados ao desenvolvimento da DPP. Aplicaram-se dois questionários, com o perfil socioeconômico e a Escala de Depressão Pós-Parto de Edimburgo, visando rastrear mães com sintomas compatíveis com DPP. Pontuações maiores ou iguais a 10 foram consideradas positivas e encaminhadas para avaliação com profissional especializado. Os dados foram avaliados estatisticamente pelo programa SPSS 10.0. Analisaramse 146 mães, com média etária de 28,97 anos e período de pós-parto majoritário de 3-6 meses. Houve predomínio de mães casadas, com ensino superior e renda de 4-6 salários mínimos. Um total de $31,5 \%$ das mães apresentou escore compatível com DPP, sem pico de incidência em relação ao período de pósparto. Relacionamento insatisfatório mostrou-se como fator de risco para DPP e ajuda insatisfatória como possível fator. Idade e escolaridade maternas, estado civil e renda, não mostraram relevância estatística. Encontrou-se média de casos compatíveis com DPP acima da descrita na literatura, entretanto, tal média apresenta-se com grande variação, evidenciado o caráter cultural e ambiental do transtorno.

DESCRITORES: Depressão pós-parto; Fatores de risco; Incidência, Estudos transversais.
ABSTRACT: Postpartum psychiatric illnesses were recognized as specific disorder recently. Therefore, they are under-researched and there's few knowledge about them. However, the diagnosis is important due to morbidity and frequency that affects the mothers. The prevalence of postpartum depression (PPD) is $10-15 \%$, with studies pointing to $22 \%$. Its etiology is multifactorial and includes biological, psychological and social factors, and may last up to one year after delivery. We sought to evaluate the incidence of PPD in mothers during the first year postpartum in Curitiba - PR, still trying to identify mood changes occurring in the postpartum period and the main risk factors associated with the development of PPD. We applied two questionnaires, the socioeconomic profile and Edinburgh Postnatal Depression Scale, aiming to track mothers with symptoms compatible with PPD. Scores greater than or equal to 10 were considered positive and referred for evaluation with a specialized professional. The data were statistically analyzed by SPSS 10.0. We analyzed 146 mothers with a mean age of 28.97 years and the postpartum period majority of 3-6 months. There was a predominance of married mothers with higher education and income of 6.4 minimum wages. A total of $31.5 \%$ of mothers had scores compatible with PPD, without peak regarding the period after delivery. Unsatisfactory relationship proved to be a risk factor for PPD and helps unsatisfactory as a possible factor. Age and maternal education, marital status and income showed no statistical significance. It's was found an average of DPPcompatible cases above the described in the literature, however, this average is presented with great variation, indicating the cultural and environmental character of the disorder.

KEYWORDS: Depression, postpartum; Risk factors; Incidence; Cross-sectional studies.

Trabalho premiado em 1ํ lugar na categoria Epidemiologia e Saúde, no VI Encontro de Saúde Coletiva, Curitiba, PR, 10 nov. 2009.

1. Acadêmica de Medicina, Universidade Positivo, Curitiba, PR.

2. Doutora em Medicina Interna, professora adjunta da Disciplina de Clínica Médica Ambulatorial da Universidade Federal do Paraná e de Saúde da Família da Universidade Positivo, Curitiba, PR.

Endereço para correspondência: Válery Baggio Hess. Rua Castro, 699 - Curitiba, PR. E-mail: valerybaggio@uol.com.br 


\section{INTRODUÇÃO}

s doenças psiquiátricas do pós-parto
foram reconhecidas como transtorno
específico há pouco tempo ${ }^{1}$. São, por isso, pouco pesquisadas e de escasso conhecimento. Contudo, mostram-se de identificação importante pela morbidade e frequência com que acometem as puérperas.

A prevalência de depressão pós-parto (DPP) é de $10-15 \% \%^{2,3,4}$, com estudos apontando para $22 \%{ }^{5}$. Já o risco de recorrência é de $50 \%$ e aproxima-se dos $100 \%$ em mulheres com histórico de transtorno de humor e DPP prévios ${ }^{4,6}$. Sua etiologia é multifatorial e inclui fatores biológicos, psicológicos e sociais, podendo ocorrer até um ano após o parto ${ }^{6}$.

Assim sendo, o objetivo do presente estudo é avaliar a incidência de DPP em puérperas no primeiro ano de pós-parto na cidade de Curitiba - PR, tentando ainda identificar as mudanças de humor ocorridas no pós-parto e os principais fatores de risco associados ao desenvolvimento da DPP, encaminhando para tratamento específico as puérperas com fatores de risco e/ou suspeita do transtorno.

O tema foi escolhido devido à alta incidência e pela relevância como problema de saúde pública, merecendo especial cuidado por parte dos profissionais que atuam na atenção primária à saúde por ser uma doença que afeta de maneira importante a saúde materna e o desenvolvimento da criança. 0 diagnóstico precoce e a instituição do tratamento adequado diminuiriam em muito as complicações decorrentes da DPP.

\section{CASUÍSTICA E MÉTODOS}

Realizou-se um estudo descritivo transversal baseado em um questionário de identificação e no questionário auto-aplicativo Edinburgh Postnatal Depression Scale (EPDS), o qual foi elaborado em 1987 e validado no Brasil em 20007.

Todas as participantes assinaram o termo de consentimento livre e esclarecido. As mães rastreadas que obtiveram pontuação maior ou igual a 10 no EPDS foram encaminhadas para melhor avaliação com um médico especialista.

Os critérios de inclusão foram mães moradoras e cujos filhos nasceram em Curitiba, com idade de até um ano, e que se encontravam nas Unidades Básicas de Saúde de Curitiba na campanha de vacinação do dia 19 de setembro de 2009.

Os critérios de exclusão foram recusar-se a responder os questionários, responder os questionários de forma incompleta, não apresentar condições cognitivas mínimas para o entendimento do questionário aplicado e ter o diagnóstico prévio de alguma doença psiquiátrica.

A amostra foi composta por 146 mães. Os dados coletados foram divididos em categorias de análise, com cruzamento de informações e posterior aplicação do programa estatístico SPSS versão 10.0. Para a análise das variáveis contínuas, calculou-se média, mediana e desvio padrão. A análise das variáveis categóricas fez-se pela aplicação do teste de Qui-quadrado.

A coleta de dados se deu após a aprovação pelo Comitê de Ética em Pesquisa da Universidade Positivo e pelo Comitê de Ética em Pesquisa da Prefeitura Municipal de Curitiba e após o treinamento das entrevistadoras. A escolha das Unidades Básicas de Saúde envolveu seis dos nove Distritos Sanitários de Curitiba e foi realizada mediante sorteio.

\section{RESULTADOS}

Um total de 46 mães recusou-se a responder os questionários, a maioria por não querer responder $(43,18 \%)$, seguida daquelas com transtornos de humor prévio $(20,45 \%)$.

A amostra foi composta por 146 mães, com uma média etária de 28,97 anos. O período pósparto majoritário foi de 3-6 meses, correspondendo a $32,88 \%$. Houve predomínio de mães casadas $(76,03 \%)$, com ensino superior $(56,16 \%)$ e renda de 4-6 salários mínimos (31,51\%).

Dos 146 questionários coletados, 46 apresentaram escore compatível com DPP (maior ou igual a 10 pontos), totalizando uma média de $31,5 \%$ de casos compatíveis com DPP, sem predomínio em relação ao período pós-parto, conforme mostra a Tabela 1.

Tabela 1. Freqüência em relação ao período pós parto

\begin{tabular}{l|c|c|c}
\hline PERÍODO PÓS PARTO & EPDS $<10$ & EPDS $\geq 10$ & TOTAL \\
\hline $0-2$ meses & 26 mães / $81,3 \%$ & 6 mães / 18,8\% & 32 mães \\
\hline $3-6$ meses & 31 mães / 64,6\% & 17 mães $/ 35,4 \%$ & 48 mães \\
\hline $7-9$ meses & 24 mães $/ 64,9 \%$ & 13 mães $/ 35,1 \%$ & 37 mães \\
\hline $10-12$ meses & 19 mães $/ 65,5 \%$ & 10 mães $/ 34,5 \%$ & 29 mães \\
\hline TOTAL & 100 mães / 68,5\% & 46 mães $/ 31,5 \%$ & 146 mães \\
\hline
\end{tabular}


Os dados observados ainda permitem inferir que o relacionamento insatisfatório com o companheiro foi um fator de risco para DPP, com $p=0,004$ (Tabela 2).

A ajuda insatisfatória do companheiro mostrou-se, ainda, como um possível fator de risco para DPP, com $p=0,05$ (Tabela 3).

Outros dados analisados, como idade, estado civil, escolaridade, renda, se estava empregada ou não no momento e transtorno de humor prévio, não mostraram relevância estatística.

Tabela 2. Relacionamento como fator de risco

\begin{tabular}{c|c|c|c}
\hline Relacionamento & EPDS $<10$ & EPDS $\geq 10$ & TOTAL \\
\hline Satisfatório & 96 mães $/ 72,2 \%$ & 37 mães $/ 27,8 \%$ & 133 mães \\
\hline Insatisfatório & 4 mães $/ 30,8 \%$ & 9 mães $/ 69,2 \%$ & 13 mães \\
\hline
\end{tabular}

Tabela 3. Ajuda como fator de risco

\begin{tabular}{l|c|c|c}
\multicolumn{1}{c|}{ Ajuda } & EPDS $<10$ & EPDS $\geq 10$ & TOTAL \\
\hline Satisfatória & 89 mães $/ 71,8 \%$ & 35 mães $/ 28,2 \%$ & 124 mães \\
\hline Insatisfatória & 11 mães $/ 50 \%$ & 11 mães $/ 50 \%$ & 22 mães \\
\hline
\end{tabular}

\section{DISCUSSÃO}

A gestação e o puerpério são períodos de transição importantes na vida da mulher e que precisam ser avaliados com especial atenção, pois envolvem grandes mudanças que podem refletir de maneira direta na saúde mental desta.

Já na infância, a menina cresce sendo educada para ser mãe. Tal criação visa o bom desempenho e o despertar para a maternidade, transmitindo para a mulher uma imagem idealizada, a qual nem sempre é vivenciada após o nascimento de seu filho. Assim, o conflito entre o esperado culturalmente e a mudança bio-psico-social evidente podem influenciar um transtorno mental ${ }^{8}$.

Dessa forma, o período gravídico-puerperal constitui-se na fase de maior prevalência de transtornos mentais na mulher, principalmente no primeiro e no terceiro trimestres de gestação e nos primeiros 30 dias de puerpério ${ }^{9}$. Quase $30 \%$ das mulheres têm dificuldades significativas em se ajustar ao pós-parto ${ }^{10}$ e aproximadamente uma em cada 10 mulheres apresenta sintomas depressivos durante algum trimestre da gestação ou dentro do primeiro ano de pós-parto ${ }^{11}$.

A prevalência da DPP varia muito na literatura. Essas variações devem-se, provavelmente, ao uso de critérios diagnósticos e métodos diversos, bem como a diferenças econômicas e culturais entre os diversos grupos estudados ${ }^{12}$. A variação da prevalência chega a valores entre $5,92-27,5 \%{ }^{4}$. A média fica entre $10-15 \%$ das mulheres no pós-parto ${ }^{13-16}$, sendo relativamente maior nos países de baixa renda. Também tende a aumentar em casos de gestação de alto risco ${ }^{4,9}$.

No presente estudo encontrou-se uma média de $31,5 \%$ de casos compatíveis com DPP, valor acima da média descrita. Entretanto, um recente estudo apresentado no Congresso Brasileiro de Pediatria e com metodologia similar encontrou $24,46 \%$ de DPP para Ceilândia, DF ${ }^{17}$. Também outros estudos realizados no Brasil mostram taxas de prevalência variando entre $12-37,1 \%{ }^{18}$. Particularmente, algumas das prevalências encontradas são $12 \%$ no Rio de Janeiro ${ }^{19}, 13,4 \%$ em Brasília ${ }^{7}, 19,1 \%$ em Pelotas ${ }^{4}$, $20,7 \%$ em Porto Alegre ${ }^{12}, 26,9 \%$ em Belo Horizonte ${ }^{20}$, $37,1 \%$ em São Paulo ${ }^{2}$ e $39,4 \%$ em Vitória ${ }^{20}$, o que têm confirmado a prevalência muito variada e evidenciado o caráter cultural e ambiental do transtorno ${ }^{17}$.

A literatura também varia no que tange ao período adequado para a avaliação das puérperas. Alguns estudos têm sua abordagem nos três primeiros trimestres pós-parto ${ }^{5,21}$, enquanto outros abordam a partir da $12^{\mathrm{a}}$ semana pós-parto ${ }^{2}$. Outras fontes defendem seu aparecimento, na maioria dos casos, nas primeiras quatro semanas após o parto ${ }^{12}$, alcançando sua intensidade máxima nos seis primeiros meses ${ }^{4}$. Também há fontes em que se defende seu surgimento em qualquer momento dentro do primeiro ano após o parto, sendo mais frequente entre 4-8 semanas $^{22,23}$. Dentro desse panorama, o presente trabalho abrangeu todo o primeiro ano puerperal. 
Contudo, nossos achados não apontaram um pico de incidência em relação ao período pós-parto.

Existem várias escalas disponíveis para o rastreamento da DPP, mas o melhor momento para utilizá-las e sua aplicabilidade em populações multiculturais ainda não estão estabelecidas ${ }^{24}$. Dentre as mais amplamente usadas estão a Edinburgh Postnatal Depression Scale (EPDS) e a Postpartum Depression Screening Scale (PDSS) ${ }^{9,13}$.

O EPDS foi criado em 1987 e validado no Brasil por Santos et al em 20007. Foi o primeiro instrumento encontrado na literatura desenvolvido para triar especificamente a DPP. É um instrumento de auto-registro que contém 10 questões de sintomas comuns de DPP e que utiliza o formato de resposta do tipo múltipla escolha no qual a mãe escolhe as respostas que melhor descrevem o modo como ela tem se sentido na última semana. As respostas são cotadas de 0-3 de acordo com a gravidade crescente dos sintomas. O melhor ponto de corte para rastreamento é de 10 pontos ou mais (sensibilidade de $82,6 \%$ e especificidade de $65,4 \%)^{7,20}$. É de fácil utilização até mesmo para não médicos, o que viabiliza sua utilização em serviços de atenção primária à saúde ${ }^{25-27}$.

O EPDS foi originalmente construído como um instrumento de rastreamento para DPP, mas os autores da escala e outros autores propõem que, usando-se um ponto de corte maior ou igual a 13 pontos, a escala tem um alto valor preditivo positivo para o diagnóstico da doença ${ }^{7,27}$.

Em um estudo comparando a eficácia entre três métodos de diagnóstico de DPP (EPDS, PDSS e PHQ-9 - Patient Health Questionnaire), evidenciou-se que o EPDS é melhor por ser mais curto, facilmente utilizado, traduzido em várias línguas e possível para investigação em diferentes níveis socioeconômicos e etnias ${ }^{27,28}$.

Os fatores de risco associados à DPP incluem gestação não planejada, pouca idade materna, baixo nível sócio-econômico, grande número de filhos, o fato de a mãe não estar casada, relacionamento conjugal prejudicado, ajuda insatisfatória nos cuidados com a criança, desemprego, baixo peso ao nascer, alimentação do recém-nascido direto da mamadeira e doenças psiquiátricas anteriores ou durante a gestação $0^{2,9,10}$. Estudos prévios encontraram que qualquer história prévia de depressão é o maior fator de risco para o desenvolvimento da DPP ${ }^{24}$.

Nossos achados econômicos e psicossociais não mostraram relevância estatística para a maioria dos fatores pesquisados, exceto no que se refere ao relacionamento com o companheiro, que se confirmou como fator de risco, e a ajuda no cuidado com o bebê, provável fator de risco $(p=0,05)$. Atribui-se esse último achado ao tamanho da amostra e esperase que, com a ampliação desta, esse fator de risco possa ser confirmado ou afastado, assim como outros fatores pesquisados.

O quadro clínico da DPP surge de forma insidiosa e, na maioria das vezes, nas duas primeiras semanas após o parto, podendo variar na apresentação e na intensidade dos sintomas de acordo com a personalidade, a história de vida da puérpera e as mudanças bioquímicas ocorridas logo após o parto $^{20}$. Algumas mulheres costumam não reportar seus sintomas depressivos com medo do estigma associado à doença ${ }^{29}$.

De acordo com os critérios do Diagnostic and Statistical Manual of Mental Disorders (DSMIV), em geral, o quadro clínico da DPP não difere daquele apresentado por pacientes com episódios de alteração do humor extra-puerpério. $O$ início nas quatro primeiras semanas do pós-parto é apenas um especificador, que pode ser aplicado aos vários transtornos do humor. O episódio depressivo maior, no entanto, define-se pela presença de sintomas específicos por um período mínimo de duas semanas, havendo obrigatoriedade da existência de humor depressivo ou diminuição/perda do interesse nas atividades anteriormente agradáveis, associado a quatro dos seguintes: mudança significativa do peso ou do apetite, insônia ou sono excessivo, fadiga, agitação ou retardo psicomotor, sentimentos de desvalia ou culpa, perda de concentração e idéias de morte ou suicídio ${ }^{22,25,30}$.

Cursa ainda com sentimento de incapacidade em relação à maternidade e queixas psicossomáticas como cefaléia, dores nas costas, erupções vaginais e dor abdominal, sem causa orgânica aparente ${ }^{13,20,25,31}$.

Circunstâncias relativas à saúde física, à experiência emocional do parto e ao primeiro contato com o bebê predizem a sintomatologia depressiva na primeira semana do puerpério. A sintomatologia depressiva na primeira semana após o parto e a experiência emocional negativa do parto predizem a sintomatologia depressiva meses após o parto ${ }^{32}$.

A depressão puerperal afeta não só a mãe, mas também o bebê e até mesmo o pai, tendo em vista a influência desse quadro no contexto familiar. Pode se manifestar com intensidade variável, tornando-se um fator que dificulta o estabelecimento do vínculo afetivo favorável entre a mãe e o filho, podendo interferir na qualidade dos laços emocionais futuros. Há evidências de associação entre a DPP e o prejuízo no desenvolvimento cognitivo, emocional e social da criança ${ }^{19,24,31}$.

O infanticídio e o suicídio estão entre as complicações mais graves decorrentes de transtornos 
puerperais sem intervenção adequada. Os riscos de depressão não tratada incluem, ainda, pobre vínculo com o bebê, falta de auto-atendimento e abandono infantili33.

O manejo da DPP inclui programas psicoeducacionais, psicoterapia e medicamentos. A análise do risco-benefício quanto às diversas opções terapêuticas no pós-parto deve ser discutida com a mãe. A maioria das mulheres com DPP é tratada na atenção primária à saúde, com apenas alguns casos mais severos sendo referidos a um serviço de atenção secundária ${ }^{15,31}$. Algumas pesquisas sugerem que a DPP pode ser tratada com as mesmas intervenções utilizadas nas depressões que ocorrem em outras épocas da vida, mas existem poucos estudos

\section{REFERÊNCIAS}

1. Kaplan HI, Sadock BJ. Síndromes psiquiátricas pósparto. In: Kaplan H, Sadock B, organizadores. Tratado de psiquiatria. 7a ed. Porto Alegre: Artmed; 1997. p.1134-41.

2. Schmidt EB, Piccoloto NM, Muller MC. Depressão pós-parto: fatores de risco e repercussões no desenvolvimento infantil. Psico USF. 2005;10(1):61-8.

3. Camacho RS, Cantinelli FS, Ribeiro CS, Cantilino A, Gonsales BK, Braguittoni E, Rennó J. Transtornos psiquiátricos na gestação e no puerpério: classificação, diagnóstico e tratamento. Rev Psiq Clin. 2006;33(2):92102.

4. Cruz EBS, Simões GL, Cury AF. Rastreamento da depressão pós-parto em mulheres atendidas pelo Programa Saúde da Família. Rev Bras Ginecol Obstet. 2005;27(4):181-8.

5. Mallikarjun PK, Oyebode F. Prevention of postnatal depression. J R Soc Promotion Health. 2005;125:2216.

6. Moraes IGS, Pinheiro RT, Silva RA, Horta BL, Sousa $P R L$, Faria AD. Prevalência da depressão pós-parto e fatores associados. Rev Saúde Pública. 2006;40(1):6570.

7. Gjerdingen D, Crow S, McGovern P, Miner M, Center B. Postpartum depression screening at well-child visits: validity of a 2-question wcreen and the PHQ-9. Ann Fam Med. 2009;7:63-70.

8. Azevedo KR, Arrais AR. O mito da mãe exclusiva e seu impacto na depressão pós-parto. Psicol. Reflexão Crítica. 2006;19(2):269-76.

9. Pereira PK, Lovisi GM. Prevalência da depressão gestacional e fatores associados. Rev Psiquiatr Clin. 2008;35(4):144-53.

10. Silva E, Botti N. Depressão puerperal: uma revisão de literatura. Rev Eletrônica Enferm [serial online];7(2):231-8. Disponível em: http://www.fen.ufg.br.

11. Costa R, Pacheco A, Figueiredo B. Prevalência e randomizados para guiar a prática nessa população específica $^{24,31}$.

\section{CONCLUSÃO}

A taxa de DPP encontrada foi mais alta do que a descrita na literatura, entretanto, o teste utilizado para rastreamento apresenta alta sensibilidade (82\%), podendo incluir resultados falso-positivos. Por essa razão e pelo alto prejuízo ocasionado por essa doença à saúde materno-infantil e interação familiar, as mulheres com resultado positivo foram encaminhadas para avaliação com um médico especializado para esclarecimento diagnóstico e tratamento do transtorno, se necessário. preditores de sintomatologia depressiva após o parto. Rev Psiquiatr Clin. 2007;34(4):157-65.

12. Zambaldi C, Cantilino A, Sougey B. Sintomas obsessivo-compulsivos na depressão pós-parto: relato de caso. Rev Psiquiatr. 2008;30(2)155-8.

13. Santos IS, Matijasevish A, Tavares BF, Barros AJD, Botelho IP, Lapolli C, Magalhães PVS, Barbosa APPN, Barros FC. Validation of the Edinburgh Postnatal Depression Scale (EPDS) in a sample of mothers from the 2004 Pelotas Birth Cohort Study. Cad Saúde Pública. 2007;23(11):2577-88.

14. Faisal-Cury A, Tedesco JJ, Kahhale S, Menezes PR, Zugaib M. Postpartum depression: in relation to life events and patterns of coping. Arch Women Ment Health. 2004;7(2):123-31.

15. Felix GMA, Gomes APR, França PS. Depressão no ciclo gravídico-puerperal. Com Ciên Saúde. 2008;19(1):51-60.

16. Silva D, Souza M, Moreira V, Genestra M. Depressão Pós-Parto: Prevenção e Conseqüências. Rev MalEstar Subjetividade. 2003;439-50.

17. Ruschi GEC, Sun SY, Mattar R, Filho AC, Zandonade E, Lima VJ. Aspectos epidemiológicos da depressão pós-parto em amostra brasileira. Rev Psiq Rio Grande Sul. 2007;29(3)274-80.

18. Stewart DE, Robertson E, Dennis CL, Grace S. An evidence-based approach to postpartum depression. World Psychiatry. 2004;3(2):97-8.

19. Marcus SM. Depression during pregnancy: rates, risks and consequences. Can J Clin Pharmacol. 2009;16(1):15-22.

20. Figueira P, Corrêa H, Diniz LM, Silva MAR. Edinburgh Postnatal Depression Scale for screening in the public health system. Rev Saúde Pública. 2009;43(1):1-5.

21. Chew-Graham CA, Sharp D, Chamberlain E, Folkes L, Turner KM. Disclosure of symptoms of postnatal depression, the perspectives of health professionals 
and women: a qualitative study. BMC Family Pract. 2009;10(7)1-9.

22. Tannous L, Gigante LP, Fuchs SC, Busnello EDA. Postnatal depression in southern Brazil: prevalence and its demographic and socioeconomic determinants. BMC Psychiatry. 2008;8(1)1-8.

23. Horowitz JA, Murphy CA, Gregory KE, Wojcik J. Community-based postpartum depression screening: results from the CARE Study. Psychiatr Serv. 2009;60(11):1432-4.

24. Dietz PM, Williams SB, Callaghan WM, Bachman DJ, Whitlock EP, Hornbrook MC. Clinically identified maternal depression before, during and after pregnancies Ending in live births. Am J Psychiatry. 2007;164(10):1515-20.

25. Payne JL. Antidepressant use in the postpartum period: practical considerations. Am J Psychiatry. 2007;164(9):1329-32.

26. Buist A. Perinatal depression: assessment and management. Aust Fam Phys. 2006;35(9):670-73.

27. Hewitt CE, Gilbody SM, Brealey S, Paulden M, Palmer S, Mann R, et al. Methods to identify postnatal depression in primary care: an integrated evidence
Synthesis and value of information analysis. Health Technol Assessment. 2009;13(36):91-131.

28. Friedman SH, Resnick PJ. Postpartum depression: an update. Women Health (Lond Engl). 2009;5(3):28795.

29. Martins D, Pires AP. O comportamento parental de companheiros de mulheres com depressão pós-parto. Mudanças - Psicol Saúde. 2008;16(2):106-15.

30. Santos T, Nicolau LJC, Pena RN, Zaconeta ACM. Depressão pós-parto: é exequível o rastreamento pelo pediatra? In: Anais do $34^{\circ}$ Congresso Brasileiro de Pediatria, Brasílía; 2009. p.165.

32. Lusskin SI, Misri S. Postpartum blues and depression. Uptodate. 2010; Out 12. Available from: http://www.uptodate.com/onlineHanusa BH, Scholle SH, Haskett RF, Spadaro K, Wisner KL. Screening for depression in the postpartum period: a comparison of three instruments. J Womens Health. 2008;17(4):585-96. Poobalan AS, Aucott LS, Ross L, Smith WCS, Helms PJ, Williams JHG. Effects of Treating postnatal depression on mother-infant interaction and child development. Brit J Psychiatr. 2007;191:378-86.

Artigo recebido em: 03/04/2011

Artigo aceito em: 17/07/2011 